\title{
CONVOLUTION PROPERTIES ASSOCIATED WITH CERTAIN SUBCLASSES OF ANALYTIC FUNCTIONS
}

\section{ABSTRACT}

Murugusundaramoorthy and Magesh (2004) introduced the subclasses $\operatorname{TS}(\alpha, \beta)$ and $\operatorname{TS}_{b}(\alpha, \beta)$ of uniform convex functions and starlike functions with negative coefficients where they obtained some results. Our aim here is to investigate the convolution properties associated with the subclasses $T S^{*}(\alpha, \beta)$ and $\operatorname{TS}_{b}^{*}(\alpha, \beta)$ respectively by applying certain techniques based especially upon the Cauchy-Schwarz and Holder inequalities. Some consequences are also discussed.

KEY WORDS: Analytic, Convolution, Convex, Starlike, Univalent.

\section{INTRODUCTION, DEFINITIONS AND PRELIMINARY RESULTS.}

Denoted by $S$ the class of functions of the form

$$
f(z)=z+\sum_{n=2}^{\infty} a_{n} z^{n}
$$

that are analytic and univalent in the unit disk $E=\{z:|z|<1\}$.Also, ST and CV are the subclasses of $S_{n}$ that are respectively starlike and convex.

A function is uniformly convex(uniformly starlike) in $E$ if $f(z)$ is in CV(ST) and has the property that for every circular arc $\gamma$ contained in $E$, with centre $\varepsilon$ also in $E$. the arc $f(\gamma)$ is convex(starlike) with respect to $f(\varepsilon)$. The class of uniformly convex functions is denoted by UCV and the class of uniformly starlike functions by UST.

It is well known that

$$
f \in U C V \Leftrightarrow\left|\frac{z f^{\prime \prime}(z)}{f^{\prime}(z)}\right| \leq \operatorname{Re}\left\{1+\frac{z f^{\prime \prime}(z)}{f^{\prime}(z)}\right\}
$$

see Ma and Minda (1992). Ronning (1993) introduced a new subclass of starlike functions related to UCV defined as

$$
f \in S_{p} \Leftrightarrow\left|\frac{z f^{\prime}(z)}{f(z)}-1\right| \leq \operatorname{Re}\left\{\frac{z f^{\prime}(z)}{f(z)}\right\} .
$$

We note here that $f(z) \in U C V \Leftrightarrow z f^{\prime}(z) \in S_{p}$.

Later, Ronning (1993) generalized the class $S_{p}$ by introducing a parameter $\alpha,-1 \leq \alpha<1$,

$$
f \in S_{p}(\alpha) \Leftrightarrow\left|\frac{z f^{\prime}(z)}{f(z)}-1\right| \leq \operatorname{Re}\left\{\frac{z f^{\prime}(z)}{f(z)}-\alpha\right\}
$$

Also Murugusundaramoorthy and Magesh (2004) introduced subclasses $\operatorname{TS}(\alpha, \beta)$ and $\operatorname{TS}_{3}(\alpha, \beta)$.

Here we let $T S(\alpha, \beta)=S(\alpha, \beta) \cap T$ where $T$, the subclass of $S$ consisting of functions of the form

$$
f(z)=z-\sum_{n=2}^{\infty} a_{n} z^{n}, a_{n} \geq 0, \forall n \geq 2 .
$$

This class of functions was introduced and studied by Silverman(1995), Silverman and Silvia (1997). And also $T S_{b}(\alpha, \beta)$ denote the class of function, $f(z)$ in $T S(\alpha, \beta)$ and be of the form

A. T. Oladipo, Department. of Pure and Applied Mathematics, Ladoke Akintola Univ. of Tech., P. M. B. 4000, Ogbomoso, Nigeria 


$$
f(z)=z-\frac{b(1-\alpha)(c)}{(2+\beta-\alpha)(a)} z^{2}-\sum_{n=3}^{\infty} a_{n} z^{n} \quad\left(a_{n} \geq 0 \quad 0 \leq b \leq 1\right) .
$$

The main aim of this paper is to investigate the convolution properties associated with the subclasses $T S^{*}(\alpha, \beta)$ and $T S_{b}^{*}(\alpha, \beta)$.

A necessary and sufficient condition for a function $f(z)$ of the form (1.2) to be in the class $\operatorname{TS}^{*}(\alpha, \beta)$, $-1 \leq \alpha<1, \beta \geq 1$ is that

$$
\sum_{n=2}^{\infty}[n(1+\beta)-(\alpha+\beta)] \frac{(a)_{n-1}}{(c)_{n-1}} a_{n} \leq 1-\alpha
$$

Also, $\operatorname{TS}_{h}{ }^{*}(\alpha, \beta)$ denote the class of functions $f(z)$ in $\operatorname{TS}^{*}(\alpha, \beta)$ and of the form

$$
f(z)=z-\frac{b(1-\alpha)(c)}{(2+\beta-\alpha)(a)} z^{2}-\sum_{n=3}^{\infty} a_{n} z^{\prime \prime} \quad\left(a_{n} \geq 0\right), 0 \leq b \leq 1
$$

if and only if

$$
\sum_{n=2}^{\infty}[n(1+\beta)-(\alpha+\beta)] \frac{(a)_{n-1}}{(c)_{n-1}} a_{n} \leq(1-b)(1-\alpha) \quad-1 \leq \alpha<1, \beta \geq 0
$$

To do this we need the following preliminary results which we shall state without proof.

Theorem A; Murugusundaramoorthy and Magesh (2004). A necessary and sufficient condition for $f(z)$ of the form (1:2) to be in the class $T S(\alpha, \beta),-1 \leq \alpha<1, \beta \geq 0$ is that

$$
\sum_{n=2}^{\infty}[n(1+\beta)-(\alpha+\beta)] \frac{(a)_{n-1}}{(c)_{n-1}} a_{n} \leq 1-\alpha
$$

Theorem $B$; Murugusundaramoorthy and Magesh (2004). Let function $f(z)$ be defined by (1.3) then $f(z) \in \operatorname{TS}_{b}(\alpha, \beta)$ if and only if

$$
\sum_{n=3}^{\infty}[n(1+\beta)-(\alpha+\beta)] \frac{(a)_{n-1}}{(c)_{n-1}} a_{n} \leq(1-b)(1-\alpha)
$$

Finally, for functions $f_{i}(z) \in S \quad(j=1, \ldots, m)$ given by

$$
f_{,}(z)=z-\sum_{n=2}^{\infty} a_{n, 1} z^{n} \quad(j=1, \ldots . . m)
$$

the Hadamard product(or convolution) is defined by

$$
\left(f_{1}^{*} \ldots f_{n} \dot{X}(z)=z-\sum_{n=2}^{\infty}\left(\prod_{i=1}^{m} a_{n, 1}\right) z^{n}\right.
$$

\section{CONVOLUTION PROPERTIES}

Theorem 2.1; If $f,(z) \in T S^{*}(\alpha, \beta) \quad(j=1, \ldots, m)$, then $\left(f_{1}^{*} \ldots f_{m}\right)(z) \in T S^{*}(\rho, \beta)$

where

$$
\rho=1-\frac{(n-1)(c)_{n-1} \prod_{i=1}^{m}\left(1-\alpha_{1}\right)}{(a)_{n-1} \prod_{i=1}^{m}\left[n(1+\beta)-\left(\alpha_{i}+\beta\right)\right]-(c)_{n-1} \prod_{j=1}^{m}\left(1-\alpha_{1}\right)}
$$


The result is sharp for the functions $f_{i}(z)$ given by

$$
f,(z)=z-\left(\frac{1-\alpha_{1}}{(c)_{n-1}}\right) z^{n}
$$

Proofi; Following the work of Owa [1,2 (1992)], Owa and Srivastava (2003), we use the principle of mathematical induction in our proof of Theorem2.1.

Let $f_{1}(z) \in T S^{\circ}\left(\alpha_{1}, \beta\right)$ and $f_{2}(z) \in T S^{*}\left(\alpha_{2}, \beta\right)$. Then the inequality

$$
\sum_{n=2}^{\infty}[n(1+\beta)-(\alpha,+\beta)] \frac{(\alpha)_{n-1}}{(c)_{n-1}} a_{n, 1} \leq 1-\alpha_{1}
$$

that is, for $m=1$, we see that $\rho=\alpha_{1}$. For $m=2$ Theorem A gives

$$
\sum_{n=2}^{\infty} \sqrt{\frac{n(1+\beta)-(\alpha,+\beta)}{1-\alpha_{1}} \frac{(a)_{n-1}}{(c)_{n-1}} a_{n, 1}} \leq 1 \quad(j=1,2)
$$

Thus by applying the Cauchy-Schwarz inequality we have

$$
\begin{aligned}
& \left|\sum_{n=2}^{\infty} \sqrt{\frac{\left[n(1+\beta)-\left(\alpha_{1}+\beta\right)\left[n(1+\beta)-\left(\alpha_{2}+\beta\right)\right](a)_{n-1}\right.}{(c)_{n-1}}\left(a_{n, 1}\right)\left(a_{n, 2}\right)}\right|^{2} \leq \\
& \left(\sum_{n=2}^{\infty} \frac{n(1+\beta)-\left(\alpha_{1}\right)\left(1-\alpha_{2}\right)}{1-\alpha_{1}} a_{n . i}\right)\left(\sum_{n=2}^{\infty} \frac{n(1+\beta)-\left(\alpha_{2}+\beta\right)}{1-\alpha_{2}} a_{n .2}\right) \frac{(n)_{n-1}}{(c)_{n-1}} \leq 1
\end{aligned}
$$

Therefore, if

$$
\sum_{n \rightarrow \infty}^{\infty} \frac{n-\delta}{1-\delta}\left(a_{n, 1}\right)\left(a_{n, 2}\right) \leq \sum_{n=2}^{\infty} \sqrt{\frac{\left[n(1+\beta) \cdots\left(\alpha_{1}+\beta\right)\left[n(1+\beta)-\left(a_{2}+\beta\right)\right](a)_{n-1}\right.}{\left(1-a_{1}\right)\left(1-\alpha_{2}\right)}\left(a_{n, 1}\right)\left(a_{n, 2}\right)}
$$

that is, if

$$
\sqrt{\left(a_{n, 1}\right)\left(a_{n, 2}\right)} \leq \frac{1-\delta}{n-\delta} \sqrt{\frac{\left.\left[m(1+\beta)-\left(\alpha_{1}+\beta\right)\right] n(1+\beta)-\left(\alpha_{2}+\beta\right)\right](\alpha)_{n-1}}{\left(1-\alpha_{1}\right)\left(1-\alpha_{2}\right)}}
$$

then, $\left(f_{1}^{*} f_{2}\right)(z) \in T S^{*}(\delta, \beta)$

We also note that the inequality (2.3) yields

$$
\sqrt{a_{n, 1}} \leq \sqrt{\frac{1-\alpha \alpha_{i}}{n(1+\beta) \ldots(\alpha,+\beta)(a)_{n-1}}} \quad(n=2.3 \ldots, j=1.2)
$$

Consequently, if 
$\sqrt{\frac{\left(1-\alpha_{1}\right)\left(1-\alpha_{2}\right)}{\left[n(1+\beta)-\left(\alpha_{1}+\beta\right)[n(1+\beta)-(\alpha+\beta)] \frac{(c)_{n-1}}{(a)_{n-1}}\right.}} \leq \frac{1-\delta}{n-\delta} \sqrt{\frac{\left[n(1+\beta)-\left(\alpha_{1}+\beta\right)\left[n(1+\beta)-\left(\alpha_{2}+\beta\right)\right]\right.}{\left(1-\alpha_{1}\right)\left(1-\alpha_{2}\right)} \frac{(a)_{n-1}}{(c)_{n-1}}}$ That is, if

$$
\frac{n-\delta}{1-\delta} \leq \frac{\left[n(1+\beta)-\left(\alpha_{1}+\beta\right)\right]\left[n(1+\beta)-\left(\alpha_{2}+\beta\right)\right](a)_{n-1}}{\left(1-\alpha_{1}\right)\left(1-\alpha_{2}\right)} \frac{(n=2.3 \ldots)}{(c)_{n-1}}
$$

then, we have $\left(f_{1}^{*} f_{2}\right)(z) \in T S^{*}(\delta, \beta)$. It follows from (2.4) that

$$
\delta \leq 1-\frac{(n-1)(c)_{n-1}\left[\left(1-\alpha_{1}\right)\left(1-\alpha_{2}\right)\right]}{\left[n(1+\beta)-\left(\alpha_{1}+\beta\right)\left[n(1+\beta)-\left(\alpha_{2}+\beta\right)\right)(a)_{n-1}-\left(1-\alpha_{1}\right)\left(1-\alpha_{2}\right)(c)_{n-1}\right.}
$$

which shows that $\left(f_{1}^{*} f_{2}\right)(z) \in T S^{*}(\delta, \beta)$, where

$$
\delta=1-\frac{(n-1)(c)_{n-1}\left[\left(1-\alpha_{1}\right)\left(1-\alpha_{2}\right)\right]}{\left[n(1+\beta)-\left(\alpha_{1}+\beta\right)\right]\left[n(1+\beta)-\left(\alpha_{2}+\beta\right)\right)(a)_{n-1}-\left(1-\alpha_{1}\right)\left(1-\alpha_{2}\right)(c)_{n-1}}
$$

Therefore, the result is true for $m=2$

Next we suppose that the result is true for any positive integer $m$. Then we have

where

$$
\left(f_{1}^{*} f_{2} * \ldots * f_{m}\right)(z) \in T S^{*}(\tau, \beta)
$$

$$
\tau=1-\frac{(n-1)(c)_{n-1} \prod_{i=1}^{m}\left(1-\alpha_{i}\right)}{(a)_{n-1} \prod_{i=1}^{m}[n(1+\beta)-(\alpha,+\beta)]-(c)_{n-1} \prod_{i=1}^{m}\left(1-\alpha_{i}\right)}
$$

and $\rho$ is given by (2.1). After a simple calculation we obtain

$$
\rho=1-\frac{(n-1)(c)_{n-1} \prod_{l=1}^{m+1}\left(1-\alpha_{l}\right)}{(a)_{n-1} \prod_{l=1}^{m+1}\left[n(1+\beta)-\left(\alpha_{l}+\beta\right)\right]-(c)_{n-1} \prod_{l=1}^{m+1}\left(1-\alpha_{l}\right)}
$$

This shows that the result is true for $m+1$. Therefore, by mathematical induction the result is true for any positive integer $m$.

Further, taking the function $f(z)$ defined by (2.2) we have

$$
\left(f_{1}^{*} \ldots{ }^{*} f_{m}\right)(z)=z-\left\{\left(\frac{\left(1-\alpha_{1}\right)}{\left(n(1+\beta)-\left(\alpha_{1}+\beta\right)\right.} \frac{(c)_{n-1}}{(a)_{n-1}}\right)\right\} z^{n}=z-A_{n} z^{n}
$$

where

$$
A_{n}=\prod_{j=1}^{m} \frac{1-\alpha_{1}}{\left[n(1+\beta)-\left(\alpha_{1}+\beta\right)\right] \frac{(c)_{n-1}}{(a)_{n-1}}}
$$

It follows that 


$$
\sum_{n=2}^{\infty}\left(\frac{n(1+\beta)-(\rho+\beta)}{1-\rho} \frac{(a)_{n-1}}{(c)_{n-1}}\right) A_{n}=1
$$

This evidently complete the proof of Theorem2.1.

Letting $\alpha_{j}=\alpha(j=1, \ldots, m)$ in Theorem 2.1, we have

Corollary A; if $f,(z) \in T S^{*}(\alpha, \beta) \quad(j=1, \ldots, m)$, then

$$
\left(f_{1}^{*} f_{2}^{*} \ldots f_{m}\right)(z) \in T S^{*}(\rho, \beta)
$$

where

$$
\rho=1-\frac{(n-1)(c)_{n-1}(1-\alpha)^{m}}{(a)_{n-1}(n(1+\beta)-(\alpha+\beta))^{m}-(1-\alpha)^{m}(c)_{n-1}}
$$

The result is sharp for the functions $f,(z) \quad(j=i, 2, \ldots, m)$ given by

$$
f_{i}(z)=z-\left(\frac{1-\alpha}{n(1+\beta)-(\alpha+\beta)} \frac{(c)_{n-1}}{(a)_{n-1}}\right) z^{n} \quad(j=1,2, \ldots, m)
$$

Setting $\alpha=-1$ and $\beta=0$ in Corollary A to obtain

Corollary B; If $f_{,}(z) \in T S^{*}(-1,0) \quad(j=1,2, \ldots, m)$, then

$$
\left(f_{1}^{*} f_{2}^{*} \ldots * f_{m}\right)(z) \in T S^{*}(\rho, 0)
$$

where

$$
\rho=1-\frac{2^{m}(n-1)(c)_{n-1}}{(n+1)^{m}(a)_{n-1}-2^{m}(c)_{n-1}}
$$

The result is sharp for the functions $f,(z) \quad(j=1,2, \ldots, m)$ given by

$$
f_{i}(z)=z-\left(\frac{2}{n+1} \frac{(c)_{n-1}}{(a)_{n-1}}\right) z^{n} \quad(j=1.2, \ldots . m)
$$

Setting $\beta=0$ in Theorem2.1, we have

Corollary $\mathrm{C}$; If $f,(z) \in I S^{*}(\alpha, 0) \quad(j=1,2, \ldots, m)$, then

$$
\left(f_{1}^{*} f_{2}^{*} \ldots f_{m}\right)(z) \in T S^{*}(\rho .0)
$$

where

$$
\rho=1-\frac{(n-1)(c))_{n-1} \prod_{t=1}^{m}\left(1-\alpha_{l}\right)}{(a)_{n-1} \prod_{i=1}^{m}\left(n-\alpha_{l}\right)-(c)_{n-1} \prod_{i=1}^{m}\left(1-\alpha_{i}\right)}
$$

The result is sharp for the functions 
$f_{j}(z)=z-\left(\frac{1-\alpha,}{n-\alpha,} \frac{(c)_{n-1}}{(a)_{n-1}}\right) z^{n} \quad(j=1,2, \ldots, m)$

By fixing the second Coefficient, and putting $\beta=0$ we have the following

Corollary $\mathrm{D}$; If $f_{j}(z) \in T S^{*}\left(\alpha_{j}, \beta\right) \quad(j=1,2, \ldots, m)$

$$
\left(f_{1}^{*} f_{2}^{*} \ldots{ }^{*} f_{m}\right)(z) \in T S^{*}(\rho, \beta)
$$

where

$$
\rho=1-\frac{c \prod_{j=1}^{m}\left(1-\alpha_{i}\right)}{a \prod_{i=1}^{m}\left(2-\alpha_{i}\right)-c \prod_{i=1}^{m}\left(1-\alpha_{i}\right)}
$$

The result is sharp for the functions $f_{i}(z)(j=1,2, \ldots, m)$ given by

$$
f_{1}(z)=z-\left(\frac{c\left(1-\alpha_{1}\right)}{a\left(2-\alpha_{1}\right)}\right) z^{2} \quad(j=1,2, \ldots, m)
$$

After fixing second coefficient as in Theorem $B$, we have the next Theorem Theorem 2.2; if $f,(z) \in T S_{h}^{*}(\alpha, \beta) \quad(j=1,2, \ldots, m)$, then

$$
\left(f_{1}^{*} \ldots f_{m}\right)(z) \in T S_{b}^{*}(\rho, \beta)
$$

where

$$
\rho=1-\frac{(n-1)(1-b)(c)_{n-1} \prod_{j=1}^{m}\left(1-\alpha_{j}\right)}{(a)_{n-1} \prod_{j=1}^{n}\left[n(1+\beta)-\left(\alpha_{j}+\beta\right)\right]-(1-b)(c)_{n-1} \prod_{j=1}^{n}\left(1-\alpha_{j}\right)}
$$

The result is sharp for the functions $f_{l}(z) \quad(j=1,2, \ldots, m)$, given by

$$
f_{i}(z)=z-\left(\frac{(1-b)\left(1-\alpha_{1}\right)(c)_{n-1}}{n(1+\beta)-\left(\alpha_{j}+\beta\right)} \frac{(c)_{n-1}}{(a)_{n-1}}\right) z^{n} \quad(j=1,2, \ldots, m)
$$

Proof: Following the same method as in Theorem 2.1 with some simple calculation the result follows.

Letting $\alpha_{j}=\alpha(j=1,2, \ldots, m)$ in Theorem 2.2; we have

Corollary E; If $f_{i}(z) \in T S_{h}^{*}(\alpha, \beta) \quad(j=1,2, \ldots, m)$, then

$$
\left(u f^{*}{ }^{*} f_{m}\right)(z) \in T S_{b}^{*}(\rho, \beta)
$$

where 


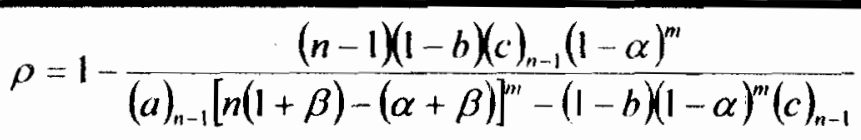

The result is sharp for the functions $f,(z) \quad(j=1,2, \ldots, m)$ given by

$$
f,(z)=z-\left(\frac{(1-b)(1-\alpha)(c)_{n-1}}{n(1+\beta)-(\alpha+\beta)(a)_{n-1}}\right) z^{\prime \prime} \quad(j=1,2 \ldots \ldots m)
$$

\section{REFERENCES}

Ma, W. and Minda, D., 1992. Uniformly convex functions, Ann. Polon. Math., 57, 165-175.

Murugusundaramoorthy, G. and Magesh, N., 2004. A new subclass of uniformly convex functions and corresponding subclass of starlike functions with fixed second coefficient. Journal of inequalities in Pure and Applied Mathematics. Vol. 5 issue 4 Article 85.

Owa, S., 1992. The quasi-Hadamard product of certain analytic functions, in Current Topics in Analytic Function Theory (H.M. Srivastava and S. Owa Editors). World Scientific Publishing Company Singapora. New Jersey. London. Hong Kong

Owa, S. and Srivastava, H. M., 2002. Some generalized convolution properties associated with certain subclasses of: analytic functions Journal of Inequalities in Pure and Applied Mathematics Vol. 3, Issue 3 Article 42.

Ronning. F., 1993. Uniformly convex functions and a corresponding class of starlike functions Pro. Amer. Math., Soe., $118,189-196$.

Silverman, H., 1995. Univalent functions with negative coefficients, Proc. Amer. Math. Soc. 51, 109-116.

Silverman, H. and Silvia, E. M., 1997. Fixed coefficients for subclasses of starlike functions, Houston J. Math., 7, 129. 136.

Srivastava, H. M and Owa, S. (Editors)., 1992. Current Topics in Analytic Function Theory, World Scientific Publishing Company Singapore, New Jersey, London, Hong Kong. 